Revue de recherche interdisciplinaire sur le genre et la sexualité

35 | 2018

Striges en tous genres

\title{
Roger Lallemand, les femmes et la morale
}

\section{Sylvie Lausberg}

\section{OpenEdition \\ Journals}

Édition électronique

URL : https://journals.openedition.org/sextant/413

DOI : $10.4000 /$ sextant.413

ISSN : 2795-8736

Éditeur

Éditions de l'Université de Bruxelles

\section{Édition imprimée}

Date de publication : 1 décembre 2018

Pagination : 163-174

ISBN : 978-2-8004-1636-6

ISSN : $1370-267 X$

\section{Référence électronique}

Sylvie Lausberg, «Roger Lallemand, les femmes et la morale », Sextant [En ligne], 35 | 2018, mis en ligne le 01 novembre 2021, consulté le 08 décembre 2021. URL : http://journals.openedition.org/ sextant/413 ; DOI : https://doi.org/10.4000/sextant.413

\section{@)}

La revue Sextant est mise à disposition selon les termes de la Licence Creative Commons Attribution Pas d'Utilisation Commerciale - Partage dans les Mêmes Conditions 4.0 International. 


\title{
Roger Lallemand, les femmes et la morale
}

\author{
Sylvie LAUSBERG
}

Le sénateur socialiste Roger Lallemand est connu comme le «père » de la loi de 1990 dépénalisant partiellement l'interruption volontaire de grossesse en Belgique. Curieusement, il ne m'a jamais été donné d'entendre que la co-autrice du texte, la libérale flamande Lucienne Herman-Michielsens, en serait la mère. La raison est plus complexe qu'il n'y paraît. Lucienne Herman-Michielsens ne peut en effet pas être considérée comme une militante féministe, alors que Roger Lallemand reste l'acteur principal de ce combat emblématique pour les droits des femmes. L'aboutissement de cette lutte législative révèle un second paradoxe : alors que l'enjeu est de délier la question de l'avortement de « la moralité publique ", l'accès à l'IVG relève davantage dans la pensée de Roger Lallemand d'une question morale que d'un droit à la santé des femmes. Cet hommage vise à articuler ces éléments, à rencontrer les contradictions apparentes qui traversent ce tournant législatif inédit dans l'histoire du pays pour faire émerger quelques aspects de la personnalité de Roger Lallemand en regard de l'évolution de la place des femmes dans la société belge.

\section{L'accès à l'interruption volontaire de grossesse : un combat emblématique, laïque et féministe}

La culture, ainsi que l'exercice chez cet homme d'Etat d'une réflexion permanente sur le droit, se rejoignent ici dans un engagement indissociable dans nos mémoires de ce combat laïque et féministe. Un combat qu'il a mené d'abord comme avocat, auprès de Willy Peers et d'autres médecins poursuivis pour avoir pratiqué des avortements, avant de le porter dans l'hémicycle avec le succès que l'on sait et ses avatars royaux, uniques dans les annales constitutionnelles.

Se référer à la loi du 3 avril 1990 qui porte son nom renvoie à un premier paradoxe qui réside dans l'écart entre la notoriété exceptionnelle de cette loi et la 
méconnaissance évidente tant de sa nature que de son contenu ; qu'il s'agisse de sa présence dans le Code pénal (article 350 et suivants) ou des conditions strictes qu'elle fixe pour octroyer une dépénalisation partielle. Ces conditions ne sont d'ailleurs ni précisément connues du grand public, ni totalement respectées par les praticiens, certaines étant inapplicables dans la pratique. En conséquence, le premier constat posé renvoie au large fossé entre la perception et les prescrits de ce qu'on a très longtemps appelé « la loi Lallemand ${ }^{1}$.

Au manque d'information publique et officielle sur l'IVG en Belgique qui a perduré jusqu'il y a peu ${ }^{2}$ s'ajoute un manque manifeste de transmission de l'histoire de la lutte pour l'accès à des avortements médicalisés et non clandestins. Cette dernière lacune en dit long sur l'invisibilité, aujourd'hui encore, des combats féminins, même lorsqu'ils sont portés par un homme de la stature de Roger Lallemand. On pourrait oser prétendre que son nom a recouvert, depuis et malgré lui, ce tabou social de la problématique de l'avortement. De fait, aujourd'hui quasiment personne ne sait qu'en Belgique l'IVG reste, dans les textes, un « délit contre l'ordre des familles et la moralité publique $»^{3}$. Au sens du législateur de 1867, les fondements du titre VII du Code pénal relatifs aux délits contre la moralité publique font appel à des valeurs qui, un siècle plus tard, n'ont plus cours. Il n'empêche, l'évolution de la régulation de l'avortement est restée bloquée pendant cent trente-trois ans, jusqu'au vote de la loi en 1990.

Les détails et détours de ce long processus législatif mettent en lumière le sens politique que le sénateur socialiste a déployé avec une persévérance qui force encore aujourd'hui l'admiration. D'autant que l'on assiste, depuis 2016, à une reprise du débat en commission de la Justice, contrecarrée par des oppositions et frilosités qui rappellent celles auxquelles Roger Lallemand et tous ceux qui travaillaient dans son ombre ou à ses côtés ont été confrontés ${ }^{4}$. Il faut le reconnaître, si, dans ses missions, le législateur peut proposer des modifications afin que la loi soit le reflet des aspirations de la population, en matière d'interruption volontaire de grossesse, cela n'a jamais été le cas. Les règles qui ont toujours cours se réfèrent à un ordre, celui des familles ; le transgresser est réputé mettre l'Etat en péril. Il faut encore souligner que l'interdiction de l'avortement dans le Code pénal de 1867 ne trouve plus son origine dans une quelconque volonté de protéger les personnes - contrairement

${ }^{1}$ S. Lausberg (dir.), Sortir l'IVG du Code pénal. Actes des colloques. Bruxelles 30.09.2017, Liège 23.11.2017, Sénat 27.01.2018, Bruxelles, Centre d'action laïque, 2018 (https://www. laicite.be/app/uploads/2018/01/sortir-1-avortement-du-code-penal-actes-des-colloques-2018. pdf).

${ }^{2}$ Il a fallu attendre avril 2017 pour que le site du SPF Santé mette clairement et précisément ces informations à la disposition du grand public ; cette modification fait suite à une question orale posée à la ministre de la Santé en novembre 2016 par la députée MR Kattrin Jadin (https:// www.jadin.be/pdf/QO\%20R\%20MdB-\%20acces\%20information\%20IVG.pdf).

${ }^{3}$ Comme l'indique le sondage d'opinion de l'Institut Kantar, de l'ulb et de l'Université d'Hasselt, réalisé à l'initiative du CAL en décembre 2017 Celui-ci a été publié le 17 avril 2018 par le Centre d'action laïque.

${ }^{4}$ S. Lausberg, L'avortement et le Code pénal en Belgique. 1867-2017, Bruxelles, Centre d'action laïque, 2017, p. 25 et s. (https://www.laicite.be/app/uploads/2017/09/1-avortement-etle-code-penal-en-belgique-1867-2017.pdf). 
à l'incrimination contenue dans l'article 317 du Code pénal de $1810^{5}$ - ou même un embryon - « la vie dès la conception » selon la doctrine catholique -, mais dans la volonté de contrôler l'usage du corps des femmes et de les empêcher de décider si elles veulent ou non des enfants, combien et avec qui. Cette dernière notion est essentielle à la compréhension de la législation sur l'avortement, mais également de son corollaire : celle, concomitante, sur le divorce dans laquelle Roger Lallemand a aussi tenu un rôle décisif.

$\mathrm{Au}$ début des années 1960, la condition des femmes évolue vers plus d'émancipation, sous la pression des mouvements féministes comme les Marie Mineur qui organisent des grèves, notamment à la FN de Herstal sous le slogan « A travail égal, salaire égal ${ }^{6}$. Une des grandes figures de l'époque, Jeanne Vervoort-Vercheval, précise que leur conscience politique marxiste s'est ouverte à une préoccupation pour la santé des femmes dictée par les conséquences épouvantable des avortements clandestins $^{7}$. A la mise sur pied d'une filière vers les Pays-Bas succède sa rencontre avec le gynécologue Willy Peers, communiste lui aussi, basé à Namur, mais qui se rendait dans la région du Centre à l'invitation des militantes pour y conscientiser d'autres médecins à la nécessité d'accueillir les femmes enceintes et de les aider. Ce spécialiste de l'accouchement sans douleur, technique qu'il avait apprise en Union soviétique, était avant tout un humaniste, qui considérait que la femme était l'égale de l'homme. Une conviction démocratique que partageait Roger Lallemand.

Quand les premiers centres de planning ont ouvert leurs portes, au début des années 1960, l'hypocrisie de la société est totale : les femmes belges avortent qu'elles soient catholiques ou non ${ }^{8}-$, les couples se débrouillent pour contrôler les naissances, mais la législation leur dénie ces droits. En effet, outre l'avortement, toute publicité pour la contraception est interdite. En 1970, le D Peers crée la Société belge pour la légalisation de l'avortement. Le 17 janvier 1973, il est inculpé pour avoir dit publiquement qu'il pratiquait des avortements et placé en détention préventive à la prison de Namur. En quelques jours, des milliers de Belges signent une pétition pour sa libération, lancée par des comités de soutien qui se créent un peu partout. André Cools apporte le soutien du parti socialiste qu'il dirige ; Roger Lallemand prendra en charge la défense du prévenu. Après 36 jours de détention préventive, la Chambre du conseil de Namur décide de son renvoi devant le Tribunal correctionnel. Alors que c'était son rôle, le Parquet n'a jamais fait fixer l'affaire qui, finalement prescrite, n'a débouché sur aucun procès. Parmi les soutiens de Peers, le chanoine Pierre de Locht prend publiquement position contre l'épiscopat. L'avocat de Peers n'oubliera pas le courage de cet homme d'Eglise au cours du combat qu'il va mener et qui ne fait alors que commencer.

${ }^{5}$ R. VAN DER MADE, « Histoire de la répression de l'avortement », Revue de droit pénal et de criminologie, 5, 1947-1948, p. 404 et s.

${ }^{6}$ Un comité pluraliste et bilingue portant ce nom est créé en 1966 sous la houlette de Monique Van Tichelen, Piet Vermeylen, Marijke Van Hemeldonck et Marthe Van de Meulebroeke.

${ }^{7}$ S. Lausberg, Sortir l'ivg du Code pénal. Actes des colloques, op. cit., p. 73-75.

${ }^{8}$ Ibid., p. 75. 


\section{Adultère, divorce : le ventre des femmes belges au cour de la législation}

A la fin des années 1960, le ventre des femmes reste un enjeu de société et son usage est toujours régulé par les Codes civil et pénal. La première proposition de dépénalisation de l'IVG a été déposée par le sénateur socialiste Willy Calewaert ${ }^{9}$. Ce dernier mène parallèlement un autre combat qui touche également à l'intimité des couples, celui d'un accès égalitaire au divorce ${ }^{10}$. Sur ces deux dossiers, on trouve dans son sillage son collègue Roger Lallemand. Le cœur de ces deux dossiers est le même : une prétention politique et judiciaire au contrôle sur le corps des femmes; la crainte principale étant de leur laisser une once de liberté sexuelle dont la conséquence potentielle serait l'introduction d'un enfant adultérin au sein de la famille.

Le Code civil de 1833, largement hérité du Code Napoléon, stipule en toutes lettres : «l'infidélité de la femme est punissable, dans tous les cas, parce qu'elle peut introduire dans la famille des enfants étrangers ; celle du mari ne l'est que lorsqu'il a entretenu sa concubine dans la maison conjugale, parce qu'alors l'outrage se joint à l'adultère " (Code civil de 1833, 6 édition). Il en va de même dans le Code pénal de 1867, dont l'article 387 fixe une peine de prison de trois mois à deux ans pour une femme « convaincue d'adultère » quand l'article 389 ne vise que « le mari convaincu d'avoir entretenu une concubine dans la maison conjugale ». Dans ce cas, la peine prévue est bien moindre : un mois à un an d'emprisonnement. La réforme des modalités de divorce en Belgique fut longue et chaotique : «En ce qui concerne le divorce pour cause déterminée, il y eut d'abord l'allègement, dans certaines circonstances, du « délai de viduité » qui imposait à la femme dont le mariage avait été dissous par la mort ou le divorce de laisser s'écouler une certaine période avant de contracter une nouvelle union (loi du 30 juin 1956). Ensuite, on supprima le délai d'attente de trois ans auquel était en principe soumis le remariage « avec son complice » de l'époux coupable d'adultère (loi du 15 mai 1972) ${ }^{11}$. Il faut attendre 1974 pour que la Ioi du 28 octobre supprime cette discrimination et rétablisse une égalité juridique des conjoints face au devoir de fidélité, avant que celle du 20 mai 1987 dépénalise enfin l'adultère. Dans la foulée de ces avancées, qui progressivement remettent en cause la subordination des femmes à l'ordre patriarcal et familial, Roger Lallemand lance le processus législatif qui a bouleversé la perception et l'acceptation du divorce ${ }^{12}$ pour aboutir à la fameuse loi de 1994. De plus, en vertu de celle-ci, le divorce pour cause déterminée permet aux époux de demander réciproquement le divorce pour « excès, sévices ou injures graves de l'un d'eux envers l'autre ». Ces excès ou sévices doivent

${ }^{9}$ Proposition de loi abrogeant les articles 351 et 353 ainsi que certaines dispositions de l'article 383 du Code pénal et remplaçant le texte de l'article 353 du même Code, Sénat, n² 280, 18 février 1971, session 1970-1971. Le sénateur W. Calewaert faisait partie de la Société belge pour la légalisation de l'avortement créée par W. Peers.

10 Proposition de loi du 10 juillet 1968 modifiant certains articles du Code civil relatifs au divorce, Doc. du Sénat 68, session extraordinaire de 1968.

11 A. Devillé et O. PAYE, «La réforme des procédures de divorce », Courrier hebdomadaire du CRISP, 1495, 1995, p. 4-5.

12 Proposition de loi Lallemand et consorts, Doc. parl. 47S0860/001, session 1989/19900, déposée au Sénat le 19 décembre 1989, co-signée par une représentant de chaque groupe parlementaire, dont Lucienne Herman-Michielsens pour le PVV. 
être intentionnels et émaner d'une personne responsable de ses actes. Le juge doit vérifier, même d'office, les faits et la gravité des injures. Sur le terrain, il s'agit d'une avancée majeure pour tous les citoyens, enfin autorisés à se séparer sans passer par des procédures interminables, mais surtout pour les personnes victimes de violences conjugales qui sont, à l'époque comme aujourd'hui, en grande majorité des femmes ${ }^{13}$.

En cas de consentement mutuel entre époux les procédures de divorce sont donc simplifiées ; la proposition élaborée par Roger Lallemand et Yvan Ylieff qui a servi de base aux travaux relatifs à la loi de 1994 se fonde « sur la présomption que les époux auront pris une décision stable antérieurement à l'engagement de la procédure. [En conséquence] le temps d'épreuve imposé par la législation actuelle n'est donc plus de rigueur. Cette nouvelle conception requiert que le divorce, qu'il soit pour cause déterminée ou par consentement mutuel, ne soit plus admis, mais prononcé ${ }^{14}$.

Si cette réforme des procédures de divorce se caractérise, selon les commentateurs juridiques, par son caractère « essentiellement pragmatique et technique, autorisant l'expression d'un large consensus, sans toutefois parvenir à étouffer les options idéologiques sous-jacentes aux regroupements partisans ${ }^{15}$, elle consacre comme légitimes non seulement l'adaptation du droit aux mœurs, mais également une forme de primauté de la décision responsable des hommes ET des femmes en ce qui concerne leurs relations intimes, sur l'ordre des familles et la moralité publique. Roger Lallemand a résumé cette évolution en ces termes : «Les changements fondamentaux ne [sont pas] insufflés par les seuls responsables politiques, mais surtout par les citoyens euxmêmes. Leurs attentes et leurs réactions, aujourd'hui, sont parties intégrantes de la réponse politique $»^{16}$.

\section{Pourquoi et comment Roger Lallemand est-il devenu « le père de la loi de $1990 »$ ?}

Fidèle parmi les fidèles de Roger Lallemand, Monique Van Tichelen ${ }^{17}$ a conservé les mémos qu'elle rédigeait à son intention lorsqu'il s'agissait de revenir sur les étapes de ce débat qui a secoué le pays. Avec l'arrestation du docteur Peers, le problème fait irruption dans l'opinion publique et dans le champ politique. La prise de position publique des évêques le 6 avril $1973^{18}$ marque le coup d'envoi de l'offensive. Le

${ }^{13}$ C. VANNESTE, La politique criminelle en matière de violences conjugales : une évaluation des pratiques judiciaires et de leurs effets en termes de récidive. Rapport final, Bruxelles, Direction opérationnelle de criminologie, Institut national de criminalistique et de criminologie, 41, mai 2016, https://nicc.fgov.be/upload/publicaties/fr-rapportcvanneste_vicmai2016.pdf.

14 Proposition de loi modifiant les procédures de divorce, 545/1-91, session extraordinaire de la Chambre des représentants 1991-1992, déposée par Y. Ylieff et consorts, le 23 juin 1992, p. 2.

15 A. Devillé et O. PAYE, « La réforme des procédures de divorce », op. cit., p. 33.

16 I. Philippon, « Réflexion politique. Paroles de sage : Entretien avec Roger Lallemand », Le Vif-L'Express, 15 mai 1998, p. 22-24.

17 Historienne et activiste féministe à l'origine du comité «A travail égal, salaire égal » en 1966. A partir de 1978, elle préside le Comité pour la suspension des poursuites en matière d'IVG. Elle est décédée le 17 février 2018.

18 ConféRence des ÉvÊQues de Belgique, Déclaration [des évêques] sur l'avortement, Bruxelles, LICAP éditions, 6 avril 1973. 
Centre d'action laïque réplique avec sa Positions laïques sur l'avortement. Réponse à la déclaration des évêques ${ }^{19}$. L'affrontement est public. Cependant, les opposants à l'IVG tiennent le haut du pavé ; PSC et CVP sont de tous les gouvernements depuis 1958 et empêchent de légiférer en la matière. Monique Van Tichelen précise : «Le sujet est particulièrement tabou dans les médias flamands dont les journalistes, qu'ils soient CVP ou catholiques de la vU, refusent systématiquement d'aborder le sujet, notamment à la BRT $»^{20}$.

Le système est grippé ; la loi est en décalage par rapport à la réalité, mais elle n'est pas appliquée. L'objectif des opposants est de provoquer un immobilisme général en encommissionnant la question. La tactique fonctionne : au sein de la Commission nationale des problèmes éthiques créée suite à l'Affaire Peers ${ }^{21}$, « l'expertise médicale vient ainsi appuyer un objectif propre au sens commun : la réduction du nombre total d'avortements, médicaux ou clandestins, objectif consensuel s'il en est. Mais, le souci des sociaux-chrétiens d'éviter à tout prix la banalisation des représentations collectives en matière d'avortement rend également cet objectif des plus conflictuels. Ce qui explique que sur le plan idéologique, les travaux de la Commission se soldent par la remise de deux textes et non pas d'un seul. Le potentiel disruptif du clivage entre laïcs et catholiques doit alors être canalisé par un nouvel encommissionnement ${ }^{22}$. Une avocate libérale flamande siège au sein de cette commission, elle se nomme Lucienne Herman-Michielsens.

L'accord de gouvernement Tindemans IV (juin 1977 - octobre 1978) précise que la solution de la question de l'avortement sera désormais du ressort du Parlement. En conséquence, les dépôts successifs de nombreuses propositions de loi - des plus libérales aux plus répressives - s'accumulent tant à la Chambre qu'au Sénat. Le problème est en réalité mis sous le boisseau car aucune n'a de chance d'être adoptée, faute de majorité parlementaire. Dans notre système, il est en effet exceptionnel qu'une quelconque proposition devienne loi sauf si un gouvernement la reprend à son compte et lui donne une majorité parlementaire. Or, en l'espèce, cette ouverture se révélait impossible. Du point de vue des défenseurs de l'accès à l'IvG, ce statu quo est jugé préférable à un texte en retrait par rapport aux revendications des femmes ou risquant d'entraver la pratique médicale de l'Ivg par aspiration du foetus (sans anesthésie générale) qui, depuis 1975, se généralise dans les centres de planning familial.

19 Centre D’action lä̈Que, Positions lä̈ques en matière d'avortement. Réponse à la déclaration des évêques, Bruxelles, Imp. Van Eeckhoudt, novembre 1973.

${ }^{20}$ M. VAn Tichelen, Synthèse manuscrite réalisée à l'intention de Roger Lallemand, manuscrit daté du 27 août 2005, conservé au CAL, 15 pages.

${ }^{21}$ Créée le 13 décembre 1974, elle se réunit du 4 février 1975 au 4 mai 1976 et comprend vint-cinq membres : douze femmes et treize hommes, treize néerlandophones et douze francophones. Cinq membres sont considérés comme libéraux, six comme socialistes et quatorze comme catholiques.

22 B. MARQues-PEREIRA, «L'interruption volontaire de grossesse », Courrier hebdomadaire $d u$ CRISP, $1127 / 1$ et 1128/2, octobre 1986. Voir aussi le Rapport de la Commission nationale pour les problèmes éthiques, Documents parlementaires Sénat, n 954, 1976-1977, 11 octobre 1976, 954 pages. 
Comment sortir de l'ornière ? D'année en année, la position des opposants à la libéralisation se raidit, notamment sous l'injonction croissante de l'Eglise catholique. En 1978, l'avocate Régine Orfinger informe ses amis activistes qu'elle-même, les docteurs Peers, Soumenkoff, Vekemans Kruyen, Hubinont et son équipe sont poursuivis par le Parquet. Roger Lallemand sera leur défenseur, assisté d'un jeune avocat, Pierre Legros ; c'est lui qui l'avait mis en contact avec le $\mathrm{D}^{\mathrm{r}}$ Peers au moment de son incarcération en 1973.

Les deux avocats défendent les médecins, mais également les femmes qui ont eu recours à une IVG. Quelques documents d'archives ${ }^{23}$ résument les circonstances et les faits incriminés :

- une jeune fille de 20 ans, orpheline de mère à 10 ans, placée en institution, enceinte de père inconnu, rejetée par son père, sans travail, sans droit au chômage, sans aide du CPAS : IVG pratiquée à neuf semaines à Saint-Pierre. Dénoncée par une « dame » chez qui elle avait trouvé un travail de servante ;

- une jeune femme de 26 ans, mère de trois enfants de deux pères différents. En avril 1980, enceinte par oubli de pilule d'un troisième homme, un des enfants qu'elle n'est pas venue chercher à l'école est emmené au poste de police ; elle explique son absence suite a une hémorragie consécutive à une IVG réalisée au «Plan F ». Elle est placée en garde à vue puis inculpée ;

- une jeune fille placée par le juge depuis qu'elle a quatre ans, sans ressources, sans CPAS ni chômage est dénoncée par la mère d'une fille hébergée dans le même home qu'elle pour une IVG réalisée à Saint-Pierre, à treize semaines de grossesse ; - une jeune femme de 20 ans, violentée par son compagnon qui veut la forcer à se prostituer, porte plainte puis se rétracte. Interrogés, le compagnon et sa mère la dénoncent pour une IVG réalisée au planning Collectif Contraception.

Ces quatre parcours de vie donnent une dimension plus précise de ce dont il s'agit dans ce débat qui, dans la presse comme ailleurs, reste limité à une bataille idéologique. Leur avocat, Roger Lallemand connaît, lui, les détails de la vie de ces jeunes femmes que la justice s'apprête à juger coupables, alors qu'elles sont victimes d'abandon et de pauvreté. Remarquons que trois sur quatre ont été dénoncées par une connaissance ; à chaque fois, la délatrice est une femme...

Cela montre à quel point la qualification de délit pénal n'est pas que symbolique et a des effets désastreux dans la réalité. Ces situations révoltantes ont certainement compté dans la pugnacité de l'avocat et dans la détermination du parlementaire qu'était Roger Lallemand. Ce dernier devra en passer par de nombreuses épreuves, d'interminables atermoiements et négociations pour arriver à libérer la société belge du joug de cette scandaleuse injustice.

Les poursuites ayant repris, Monique Van Tichelen et les membres des comités pour la dépénalisation, déjà constitués, décident de mettre « la machine en marche »; ils alertent tous leurs contacts, associations de femmes, médecins, avocats, politiques, centres de planning. Un groupe de travail se réunit à l'Union des anciens étudiants de l'UlB (UAE), rassemblant des francophones et des flamands, des laïques et également des chrétiens. Quelle stratégie adopter ? Il parait impossible de faire voter rapidement

\footnotetext{
${ }^{23}$ Conservés au CAL.
} 
une loi qui mettrait fin au délit et permettrait d'arrêter les poursuites. Au cours de la séance de travail du 19 avril 1978, Roger Lallemand lance une proposition : faire adopter un texte commun pour une suspension limitée dans le temps des articles 350 et suivants du Code pénal. Objectif : surseoir aux poursuites et avoir le temps de négocier un accord politique sur le fond. C'est encore Roger Lallemand qui suggère le nom de "Comité pour la suspension des poursuites en matière d'IVG ", ci-après dénommé CSPI, dont Monique Van Tichelen devient la présidente. Une pétition est lancée qui récolte plus de 15000 signatures individuelles et l'adhésion de nombreuses associations et groupements qui vont des syndicats aux associations culturelles, de jeunesse, etc. Grâce au CPSI, des actions de coordination, d'activisme, de réflexion et de lobbying sont continuellement mises en œuvre, jusqu'à la réussite du projet douze ans plus tard, lors du vote de la loi Lallemand - Herman-Michielsens.

\section{Bretteur au prétoire, fin négociateur en coulisses}

Roger Lallemand est un lettré. Son érudition lui procure des avantages sur les idéologues : la connaissance des textes, le respect des idées et des convictions de chacun comme la conscience de la nécessité du doute pour qui prétend œuvrer en faveur du bien commun. Ulbiste, laïque, franc-maçon et socialiste, Roger Lallemand, l'avocat des médecins et des femmes récemment poursuivis, est celui qui, pour ces différents cercles, est le mieux à même de porter le combat dans l'arène politique.

Le mouvement laïque, qui a pris publiquement position pour une dépénalisation totale ${ }^{24}$, doit en rabattre sur ses prétentions, tant le climat d'opposition et l'omnipotence des partis chrétiens pèsent sur les débats. Pour aboutir, il faut sortir de la logique de la confrontation et privilégier un dialogue ; mais avec qui ?

Alors que les inculpés se retrouvent toutes les semaines au Palais de Justice, entourés de leurs avocats, le CSPI met autour de la table des personnalités d'horizons très différents. L'objectif est de soutenir l'action politique par une opinion publique mieux informée et continuellement alarmée par des manifestations, prises de position, recherches scientifiques, communiqués, etc. Ces réunions hebdomadaires s'étalent sur plusieurs années. Le 30 juin 1983, la cour d'appel de Bruxelles, par un seul et même arrêt joignant toutes les causes dont elle était saisie, acquitte toutes les prévenues, tous les médecins et leurs collaborateurs, lourdement condamnés en première instance par des peines allant jusqu'à dix-huit mois de prison. C'est le moment que saisit le Centre d'action laïque pour réunir dans une même publication, intitulée Pour une approche pluraliste de l'avortement, laïques et chrétiens progressistes. Tous s'accordent sur les fondamentaux qui doivent présider aux décisions en matière d'avortement : il s'agit du couple et de la femme, non de l'Etat ou des médecins; les sanctions pénales doivent être supprimées, la notion de détresse est inadéquate et, last but not least, " le droit n'est pas l'expression de la morale ». Un chapitre rédigé par les chrétiens est intitulé « Une approche plus humaine de l'avortement». Le chanoine de Locht l'a

${ }^{24}$ F. Coulon, J. Geairain, P. Leleux, A. Luciani, A.-M. Moenaert, W. Peers, M. Rifflet, L. Schouters-Decroly, M. Van de Meulebroeke, W. Verougstraete, Positions lä̈ques. Pour la dépénalisation totale de l'Interruption volontaire de grossesse, Bruxelles, CAL, 1978, https://www.laicite.be/app/uploads/2017/04/Positions-Laiques-Pour-la-depenalisationtotale-de-1-interruption-de-grossesse-1978.pdf. 
répété en public à la télévision : «Ils sont nombreux ceux qui pensent qu'il serait plus dangereux de laisser continuer une telle grossesse [non désirée]. L'Eglise ce n'est pas seulement simplement une autorité masculine, ça devrait être une écoute beaucoup plus grande de ce qui se vit. L'Eglise, c'est aussi les chrétiens de la base ${ }^{25}$.

C'est avec et autour de Roger Lallemand que ce combat est mené au sein du monde politique, obligé de s'ouvrir aux voix émanant de la société civile. Ce fut toute la science politique et son humanisme qui permirent d'y arriver comme il l'exprimera dans son exposé introductif au Sénat le 7 juillet 1989: «La proposition de loi est le fruit d'une longue négociation et de longues réflexions qui ont eu lieu avec Madame Herman-Michielsens. Elle est l'aboutissement d'une réflexion de plusieurs courants de pensée. Nous avons voulu aboutir à une vision commune de la problématique de l'avortement ${ }^{26}$.

Ces quelques lignes censées « exposer les raisons qui justifient l'insistance et la détermination des auteurs à défendre leur proposition de loi » recèlent des espaces de réflexion et des trésors d'informations sous-jacentes que je vais tenter de résumer ci-après. Quelle fut la teneur des discussions avec Lucienne Herman-Michielsens ? Quel est l'objet de ces négociations ? Qui représente les « différents courants de pensée » évoqués ? Que recouvre cette «vision commune » de la problématique de l'avortement alors que la fracture est historique, pérenne et aujourd'hui toujours d'actualité, du moins sur le plan politique?

\section{Quelle fut la teneur des discussions avec Lucienne Herman-Michielsens ?}

En janvier 1978, la sénatrice libérale dépose une proposition de loi ${ }^{27}$ qui a de quoi surprendre au vu du texte qu'elle a finalement cosigné avec Roger Lallemand. Les présupposés sont les suivants : l'IVG doit être considérée, en règle générale, comme une infraction à l'ordre social, armature naturelle de la société ; l'IVG a pour conséquence de détruire une vie humaine en devenir ; le respect de la vie, seuil infranchissable que la législation d'une société civilisée se doit de protéger, n'est pas le monopole d'une seule tendance philosophique mais ressenti par chacun comme fondamental. Madame Herman-Michielsens poursuit : "Quant à savoir si l'avortement libre est une forme du droit de la femme à disposer elle-même de son propre corps, c'est là une question à laquelle il doit être répondu par la négative ». Les motifs de dépénalisation de l'IVG se limiteraient donc aux menaces graves et durables sur la santé de la femme.

La semaine suivante, Leona Detiège, mandatée par André Cools, dépose à la Chambre un texte qui prévoit purement et simplement l'abrogation des articles 350, 351, 352 et 353 du Code pénal ainsi qu'un simple article de loi précisant que

${ }^{25}$ Interview du chanoine de Locht dans l'émission télévisée « Les pieds dans le plat » intitulée " Eglise et Démocratie », réalisée par Jean-Claude Defossé et Bernard Wathelet, diffusée le 2 mai 1995, Radio-télévision belge (RTBF).

${ }^{26}$ Documents parlementaires, 247-2, session extraordinaire, Sénat, 1988, p. 5.

${ }^{27}$ Proposition de loi déposée par $\mathrm{M}^{\text {me }}$ Herman-Michielsens et consorts le 11 janvier 1978, Documents parlementaires, 267 (1977-1978), n 1, Sénat de Belgique. 
l'interruption de grossesse est un acte d'exercice de l'art médical qui relève de l'article 2 de l'arrêté royal de 1978 relatif à l'art de guérir ${ }^{28}$.

Comme précisé plus haut, l'antagonisme est total. Selon Monique Van Tichelen, Lucienne Herman-Michielsens s'est accrochée à son texte, malgré les nombreuses rencontres avec le CSPI qui lui fait visiter des centres IVG du GACEHPA ${ }^{29}$. La sénatrice reconnaît la qualité médicale et humaine du travail des plannings, mais « défendit mordicus son projet par la suite. Toutes nos tentatives restèrent vaines, jusqu'au changement de majorité de $1985 »^{30}$. Bien qu'affaibli par les résultats électoraux, le PSC-CVP a mené une coalition qui a rejeté les libéraux dans l'opposition et a porté les socialistes au gouvernement. Depuis sa création, le CSPI n'a cessé de tarauder Lucienne Herman-Michielsens. Celle-ci n'en démord pas et continue à défendre son projet notamment lors d'une conférence du Willemsfonds, qui avait adhéré au CSPI, dans le Brabant flamand. A l'issue des débats, alors que Monique Van Tichelen avait une nouvelle fois pris la parole pour souligner que la proposition de loi de la sénatrice était en décalage total avec les pratiques de terrain et l'opinion publique, l'oratrice requit une suspension de séance pour pouvoir s'entretenir avec elle en aparté : «Vous avez raison, dit la sénatrice, les chrétiens ne me rejoindront jamais sur ce texte. Dites à Roger qu'il prenne la présidence de la commission de la Justice au Sénat et je prendrai celle de la Santé publique - les commissions réunies du Sénat habilitées à traiter de la question - et que je suis prête à déposer une proposition de loi avec lui. Et Monique Van Tichelen de conclure : "C'était le grand virage, enfin!»"

Ensemble, ils ont pendant plus de quatre ans poursuivi une lutte politique dans laquelle ils ont chacun joué leur partition afin de briser l'omnipotence d'une idéologie dépassée mais jusque-là politiquement incontournable. Alliés dans l'hémicycle, les deux sénateurs deviendront de véritables amis. Cette amitié prendra fin lors du décès de Lucienne Herman-Michielsens en 1995. Très ému, Roger y prendra la parole - en néerlandais ! - , rendant hommage à cette femme d'exception qui a su dépasser ses convictions personnelles pour faire primer, sur cette question d'éthique, son devoir de juriste et de représentante politique. Sans doute est-ce cela qui a permis à ces deux figures, politiquement opposées, de se rejoindre.

\section{L'homme d'une nouvelle ère}

L'introduction du rapport des discussions à la Chambre en témoigne : la Belgique est entrée dans une ère nouvelle où le droit, « dans une démocratie, doit s'efforcer de tenir compte de toutes les conceptions morales qui ont cours dans la société. Bien loin d'être une vérité absolue et éternelle, la loi évoluera en même temps que se modifient les valeurs morales qui prévalent dans la société. Quant à la morale, c'est un ensemble de valeurs qui déterminent des individus à décider ce qu'ils doivent faire ou ce qu'ils ne doivent pas faire. Elle vise plus haut que la loi parce qu'elle n'est pas soumise aux contingences de l'efficacité. Elle est une vérité individuelle, un domaine réservé

28 Proposition de loi déposée par $\mathrm{M}^{\mathrm{me}}$ Leona Detiège, Documents parlementaires, 240 (1977-1978), $\mathrm{n}^{\circ} 1$, le 18 janvier 1978.

29 Acronyme du Groupe d'action des centres extra-hospitaliers pratiquant l'avortement.

30 M. Van Tichelen, Synthèse manuscrite, op. cit., p. 8.

31 Ibid., p. 9. 
très peu perméable au droit positif. Quand le droit tranche, la question morale reste intacte $»^{32}$.

Dans un duo qui paraissait improbable jusqu'au milieu des années 1980, les deux sénateurs ont pris en tenaille les opposants à l'avortement en axant leurs argumentations respectives sur des plans différents, comme Roger Lallemand l'a plus tard exprimé à la télévision en ces termes : « Madame Herman-Michielsens tendait à considérer l'avortement comme un fait médical ; j'étais d'un avis différent et pensais qu'au contraire il fallait traiter l'avortement comme un problème moral, de conscience morale sous la responsabilité de la femme et essentiellement de la femme ${ }^{33}$. En conclusion, affirmer que Roger Lallemand est le père de la loi sur l'IVG, c'est à la fois trop et trop peu.

En effet, la prise de position résumée ci-dessus met en lumière l'axe moteur de son action déterminante dans ce dossier comme dans d'autres : démocrate, Roger Lallemand défend avant tout le droit des sujets à une égalité de traitement, quel que soit leur sexe, leur couleur de peau, leur religion ou leur singularité, comme en attestent ses combats contre l'antisémitisme et le négationnisme, pour les droits des homosexuels, la défense des artistes ou encore la réglementation des drogues. Chez ce juriste horspair, à la fois homme du XIX ${ }^{\mathrm{e}}$ siècle par son érudition et d'une modernité intuitive grâce une empathie pétrie d'une grande humanité, le droit à l'autodétermination va de pair avec une éthique de la responsabilité individuelle, comme l'illustre aussi le débat pour le choix possible d'une euthanasie. Dans ce nouveau combat, il a une nouvelle fois pu compter sur le soutien du chanoine de Locht, avec lequel il a publié, un an avant le vote de la loi, Euthanasie. Dialogue Roger Lallemand-Pierre de Locht ${ }^{34}$. S'agissant de l'IVG, la responsabilité de la femme qui souhaite y avoir recours ne peut se dissimuler derrière des préoccupations collectives, quand bien même celles-ci seraient de salubrité publique. La pression des opposants a continué à s'exercer durant les cinq années qui séparent le tournant de 1985 du vote de la loi. Les conditions qu'elle prévoit pour une dépénalisation partielle de l'Ivg sont en réalité des concessions faites aux anti-IVG. Nul doute que Roger Lallemand aurait préféré signer un texte plus respectueux de la pratique - en conservant le délai de quinze semaines qu'il avait proposé en accord avec les médecins, plus respectueux des femmes, en reconnaissant leur droit à l'autodétermination au lieu de les inférioriser par l'obligation d'attester un « état de détresse », notion inobjectivable et infantilisante, et enfin plus conforme à son éthique de la responsabilité, supprimant les sanctions pénales pour un acte qui n'a rien à faire dans ce code régissant les crimes et délits.

Avoir accepté de signer un texte qui ne correspondait pas réellement à son souhait doit cependant encore être porté à son crédit. Le moment politique était unique ; son

${ }^{32}$ Chambre des représentants. Compte rendu analytique. Session ordinaire 1989-1990, mardi 27 mars 1990. Séances plénières. Séance du matin. Projets de loi relatif à l'interruption de grossesse (transmis par le Sénat), $\mathrm{n}^{\circ}$ 950/1 à 9. $\mathrm{M}^{\mathrm{me}}$ Onkelinx, Rapporteur, p. 661.

${ }^{33}$ Interview de Roger Lallemand dans l'émission « Les années belges », réalisée par Maurice Raymakers et Willy Estersohn, diffusée le 30 mars 2003, Radio-télévision belge (RTBF).

${ }^{34}$ R. Lallemand, et P. De Locht, Euthanasie, Bruxelles, Editions Vie ouvrière, 2001. 
pays ne pouvait rester un des derniers d'Europe à criminaliser le recours à l'IVG ${ }^{35}$. Roger Lallemand a dû porter jusqu'au bout la responsabilité d'une loi qui, fruit d'un long et pénible processus, a été une grande avancée tout en laissant symboliquement l'IVG dans le Code pénal. Conscient qu'une loi parfaite n'existe pas, Roger Lallemand a engagé le législateur à prendre en compte les oppositions et à assurer la coexistence de morales divergentes, optant résolument pour la liberté et l'autonomie de la personne.

Roger Lallemand est décédé le 20 octobre 2016. A ses funérailles, son petit-fils lui a rendu hommage en soulignant que, malgré les affres de la maladie, il a souhaité laisser la vie s'en aller naturellement, sans avoir recours à l'euthanasie, alors qu'il s'est battu durant des décennies pour que d'autres puissent faire ce choix. De la cohérence d'une conscience.

${ }^{35}$ En 1989, dans l'Union européenne, seules la Belgique, l'Espagne et l'Irlande conservaient une interdiction totale de l'avortement. L'UE ne comprenait encore ni Malte, ni Chypre. Quant aux pays de l'est européen, ils étaient encore sous régime communiste où l'avortement était libre. 